\title{
Should physical laws be unit-invariant?
}

\section{Introduction}

In a paper published in this journal in 2015 , Sally Riordan reviews a recent debate about whether fundamental constants are really "constant", or whether they may change over cosmological timescales. Her context is the impending redefinition of the kilogram and other units in terms of fundamental constants - a practice which is seen as more "objective" than defining units in terms of human artefacts. She focusses on one particular constant, the fine structure constant $(\alpha)$, for which some evidence of such a variation has been reported.

Although $\alpha$ is not one of the fundamental constants involved in the proposed redefinitions, it is linked to some which are, so that there is a clear cause for concern. One of the authors she references is Michael Duff, who presents an argument supporting his assertion that dimensionless constants are more fundamental than dimensioned ones; this argument employs various systems of so-called "natural units". [see Riordan (2015); Duff (2004)]

An interesting feature of this discussion is that not only Riordan herself, but all the papers she cites, use a formula for $\alpha$ that is valid only in certain systems of units, and would not feature in a modern physics textbook. This violates another aspect of objectivity - namely, the idea that our physical laws should be expressed in such a way that they are independent of the particular units we choose to use; they should be unit-invariant.

In this paper I investigate the place of unit-invariance in the history of physics, together with its converse, unit-dependence, which we will find is a common feature of some branches of physics, despite the fact that, as I will show in an analysis of Duff's argument, unit-dependent formulae can lead to erroneous conclusions.

The structure of the paper is therefore as follows: first, in section 2, I describe and define various terms used. I then examine the concepts of unit-invariance and unit-dependence in detail in section 3; dimensions and base quantities in section 4; change of basis in section 5; and natural units in section 6. I then examine Duff's argument in section 7.

In section 8 I try to place Duff's argument, and similar arguments concerning natural units, in the context of modern physics as a whole, by suggesting a chronological lineage for unitdependence and natural units from the late $19^{\text {th }}$ century onwards. I then examine a related argument concerning the "unification" of various concepts and quantities (section 9). Finally, in section 10, I consider unit-invariance in the context of the SI system of units, and suggest a mechanism for the longevity of unit-dependent formulae.

\section{Terminology}

A number of concepts are referred to in this paper which are either not defined in the existing literature, or are not defined consistently. I will therefore list and define them here for clarity.

The vocabulaire international de métrologie (VIM) defines several of these concepts, and it seems appropriate to adopt some of their definitions, viz.:

A quantity is a property of a phenomenon, body, or substance, where the property has a magnitude that can be expressed as a number and a reference; 
A reference can be a measurement unit, a measurement procedure, a reference material, or a combination of such;

A measurement unit is a real scalar quantity, defined and adopted by convention, with which any other quantity of the same kind can be compared to express the ratio of the two quantities as a number;

A base quantity is a quantity in a conventionally chosen subset of a given system of quantities, where no subset quantity can be expressed in terms of the others;

A base unit is a measurement unit that is adopted by convention for a base quantity;

A derived quantity is a quantity, in a system of quantities, defined in terms of the base quantities of that system;

A derived unit is a measurement unit for a derived quantity.

[taken from VIM (2008) pp 4-7]

The VIM also offers a definition of dimension, namely "expression of the dependence of a quantity on the base quantities of a system of quantities as a product of powers of factors corresponding to the base quantities, omitting any numerical factor" [ibid.]. However this is unsatisfactory in my opinion, as it does not say what the correspondence is between the base quantities and the factors. I will provide a more satisfactory definition in section 3 . The VIM also does not define fundamental quantity, so I offer my own definition, as follows: "a fundamental quantity is a quantity which is defined without reference to any other quantities". (Note that this is not the same as a base quantity, because base quantities, while not defined in terms of the other base quantities, may be defined in terms of other quantities which are not base quantities.)

There is a slight residual ambiguity in the case of the terms derived quantity and derived unit. These terms are sometimes used to contrast a quantity or unit with a base quantity or unit (as above), and sometimes to contrast it with a fundamental quantity or unit. Of course, historically the default base quantities were mass, length and time, and these were also the fundamental quantities, so there was no ambiguity. But in the proposed new SI system, the units of mass, length and time will actually be defined in terms of specific amounts of action, velocity and frequency, which would then appear to qualify as the fundamental quantities. For the purposes of this paper, I will adopt the VIM definitions for derived quantity and derived unit.

Unit-invariance is not defined anywhere in the literature as far as I have been able to ascertain. John Roche - the only author to have given this property a name, to my knowledge - calls it gauge-invariance, but I avoid this term due to its specific meaning in quantum field theory. [Roche (1998) p193]. He defines it as "preserving equality under a variation in unit size."

I would define unit-invariance as follows: a property of a physical formula such that its format does not depend on choice of units. "Format" here is understood to mean the formula conceived as an algebraic expression. Roche's definition is, in any case, slightly unsatisfactory in my opinion because "variation in unit size" makes sense only after one has 
chosen a unit, whereas I see unit-invariance as a property independent of any unit system. Also, "equality" is an odd term to use, because what really matters is the format of the equation. There is no numerical equality when one changes units; numerical equality occurs only in the case of dimensionless functions.

\section{Unit-Dependence and Unit-Invariance}

Most physical laws are written in the form of an equation linking certain variables with one another, in terms of one or more constants. These constants usually have dimensions, and hence their numerical values depend on the units chosen. Equations such as the "SUVAT" equations for motion in a uniform gravitational field link inital and final velocities $(u, v)$, distance travelled $(s)$ and time taken $(t)$, in terms of a constant acceleration $(a)$ which is often the acceleration due to gravity $(g)$. An example is the vertical distance $s$ travelled by a projectile in time $t$ with initial velocity $u$ :

$$
s=u t-\frac{1}{2} g t^{2}
$$

This equation is unit-invariant: it is independent of the system of units chosen, inasmuch as the base units can be anything we like, and the derived quantities will then have appropriate units defined in terms of the base units. So, if distance is measured in metres and time in seconds, $u$ will be in metres per second and $g$ in metres per second per second. ${ }^{1}$

If we are interested in the vertical distance travelled from rest, the equation simplifies to $s=$ $-\frac{1}{2} g t^{2}$, and if we were doing a lot of calculations we might be tempted to write in the value of $g$, in our chosen system of units - e.g. SI - so that it becomes $s=-4.9 t^{2}$. But this is no longer unit-invariant; a full statement of the equation for anyone not "in the know" needs to include the units:

$$
s=-4.9 t^{2} \text { metres if } t \text { is in seconds }
$$

The equation is now unit-dependent - its format (which now includes the number 4.9) depends on the units chosen. If instead we had chosen the imperial system of units, the number would change to approximately 16 and the qualifier added would be "feet -if $t$ is in seconds". ${ }^{2}$

While the insertion of numerical values (and hence, implicitly, units) may be advantageous for the computational stage of a calculation, this is normally only done at the very end, so that we do not lose track of the units being used, and to avoid rounding or truncation errors; if we are simply using the equation to derive another, it will be left in its most general format, namely (1).

The principle of unit-invariance is not a new idea; Fourier, in 1822, was careful to point out that the equations in his Analytical Theory of Heat depend "in no respect on the choice of the unit of length" [Fourier (1955) art. 161]. Lord Rayleigh argued in his Theory of Sound (1877)

\footnotetext{
${ }^{1}$ In fact, any two of the four quantities involved here - distance, time, velocity, acceleration - can form a basis, since any two span the 2-dimensional quantity space in use here.

${ }^{2}$ This equation also carries the implication that we are working to an uncertainty of $\pm 0.5 \%$ since we have only quoted $g$ to two significant figures, so that too should really be stated.
} 
that an equation "must retain its form unchanged, whatever may be the fundamental units by which the ... quantities are numerically expressed" [Macagno (1971) p396]. Maxwell saw this as an important principle; at the beginning of his Treatise, he says that "The equations at which we arrive must be such that a person of any nation, by substituting for the different symbols the numerical values of the quantities as measured by his own national units, would obtain a true result" [Maxwell (1873) art. 2]. R.H. Dicke made a similar statement in 1962: "It is evident that the particular values of the units of mass, length, and time employed are arbitrary and that the laws of physics must be invariant under a transformation of units:" [Dicke (1962) p2163]. ${ }_{2}^{3}$ At the end of the same decade, a committee representing the Royal Society, the Chemical Society, the Faraday Society, the Institute of Physics, and the Physical Society published a report which included the recommendation that "neither the name of a physical quantity, nor the symbol used to denote it, should imply a particular choice of unit" [Royal Society $(1969)]$.

Despite these worthy efforts, unit-invariance does not appear to have yet entered the scientific mainstream; instead, formulae which are unit-invariant are often not recognised as such, and formulae that are unit-dependent are often wrongly taken to be of universal application.

The field in which unit-dependent formulae are most common is electromagnetism. This has historical origins, namely confusion about the nature of electricity and magnetism in the $19^{\text {th }}$ century. The idea that electricity might be mechanical in origin led physicists to define it in terms of mechanical quantities. Coulomb's Law for the force between two charges stated only that the force was proportional to the product of the charges divided by the square of the distance between them; Maxwell's version of the law replaced the proportionality with equality, so that it could be written algebraically as

$$
F=\frac{q_{1} q_{2}}{r^{2}}
$$

In Maxwell's "mechanical universe" this equation can be regarded as unit-invariant, since it remains true when the symbols are replaced by magnitudes in any consistent set of mechanical units, the choice of which then determines the unit of charge; in this system charge is a derived quantity. ${ }^{4}$ By the 1930 s, the consensus was that charge was actually a separate concept, independent of mechanical quantities; hence its units could be chosen independently of those of mass, length and time. A meeting in Paris in 1932 of a Commission of the International Union of Pure and Applied Physics (IUPAP), set up the previous year "to consider the symbols, units and nomenclature used in physics" recommended the insertion of a constant $K$ in this equation, to reflect this change of status:

$$
F=\frac{q_{1} q_{2}}{K r^{2}}
$$

\footnotetext{
${ }^{3}$ Maxwell saw electricity as a derived quantity, so he expressed all mechanical and electrical quantities in terms of only three base quantities, viz. mass, length and time. Maxwell's formulae can thus be regarded as unitinvariant in such a system. Dicke (born in 1916) -probably shared this view. However, if we allow for an electrical or magnetic base quantity, these formulae become unit-dependent.

${ }^{4}$ Maxwell's unit of charge is also an obvious choice where there is no convenient measure in nature that might be adopted as a unit.
} 
[Griffiths (1932) p987]. Later this equation was "rationalised" by writing $K$ as $4 \pi \varepsilon_{0}$. A.W. Rücker described the replacement of (4) by (3) as the "suppression" of the constant $K$ and of its (unknown) dimensions. [Rücker (1889) p-108].

Unit-dependent formulae are not always clearly identified as such in the literature. It is fairly common for a statement about units to be made at the beginning of a derivation, and not mentioned again - the obvious danger with this being that a particular equation may be taken out of context. Often the statement appears only once, at the beginning of the book or paper; such statements commonly take the form of an assertion that "we are working in the X system of units". Sometimes unit-dependent formulae are quoted even without any such qualification. A case in point is the fine structure constant $(\alpha)$.

$\alpha$ is a function of several other physical constants, and is a pure number with a value of about $1 / 137$; it was introduced by Sommerfeld in 1916. It is known to particle physicists as the electromagnetic coupling constant, because of its rôle in descriptions of photon-electron interactions. The definition of $\alpha$, as given by Riordan and the other authors she cites [Riordan (2015), Duff (2004), Webb et al. (1999), Davies et al._(2002)] as well as Okun and Veneziano [Duff et al. (2002)], is as follows:

$$
\alpha=\frac{e^{2}}{\hbar c}
$$

where $e$ is the electron charge, $\hbar$ is the reduced Planck constant (i.e. Planck's constant divided by $2 \pi$ ), and $c$ is the speed of light; Sommerfeld described it as the ratio of "the velocity in the first Bohr circle" (i.e. the velocity of the ground state electron) of hydrogen to the velocity of light [Sommerfeld (1922) p213]. As a ratio of similar quantities, it is thus dimensionless.

It is easy to show that (5) is not unit-invariant by taking advantage of the fact that such a formula should give the same answer in any consistent system of units. Inserting the accepted values of the constants as found in any modern textbook $\left(e=1.602 \times 10^{-19}\right.$ coulombs, $h=$ $6.626 \times 10^{-34}$ joule seconds, $c=2.998 \times 10^{8}$ metres per second) and not forgetting the $2 \pi$, we arrive at a value that is nowhere near $1 / 137$ - in fact, some 10 orders of magnitude smaller and, if we have also kept track of the units, we will have found that it is not dimensionless either. Missing, of course, is the electric constant $\varepsilon_{0}$, also known as the permittivity of free space. Including this factor does indeed give us the value of about 1/137 for $\alpha$.

The unit-invariant definition for $\alpha$ is

$$
\alpha=\frac{e^{2}}{4 \pi \varepsilon_{0} \hbar c}
$$

and it is important to note that this can be regarded as unit-invariant, even though it is tempting to identify the symbol $\varepsilon_{0}$ with the SI system. If we wish to use the cgs electrostatic system of units, the factor $4 \pi \varepsilon_{0}$ will take the value 1 , which transforms (6) into (5). The problem I wish to highlight here is that (5) is very often quoted, even in papers as recent as

${ }^{5}$ Sommerfeld did not use the $\hbar$ symbol, however, so he defined $\alpha$ as $2 \pi e^{2} / h c$. 
those cited above (published between 1999 and 2015) as though it were of universal application: in other words, unit-invariant.

The tension between unit-dependence and unit-invariance may be viewed in terms of a similar tension between the epistemic virtues of simplicity and generality. Henry Kyburg has acknowledged this: "In the interest of simplicity and intellectual economy, we wish to employ as small (and simple) a base for our structure of physical magnitudes as possible" [Kyburg (1984) p175]. Unit-dependent formulae are simpler than unit-invariant ones, since they contain fewer symbols - as does, for instance, (5) when compared with (6). Indeed, it is this simplicity that is often advanced as a reason for adopting them.

On the other hand, unit-invariant formulae apply in a greater range of circumstances: they are more general. Prior to considerations of whether we want a simple formula or a general one, therefore, we may be tempted to put the two alternatives on an equal footing. However, for unit-dependent formulae to make sense, they must be accompanied by a statement of their range of validity, and this will probably mean that they are no longer simple. As mentioned earlier, the statement of validity often appears only once. The danger of this is that a formula may be taken out of context and assumed to be of universal, rather than limited, application.

\section{Dimensions and Base Quantities}

The use of the term "dimension" to signify a "measurable property" goes back to Descartes in 1629 [Roche (1998) -p189], and was given more quantitative significance by Fourier in the early $19^{\text {th }}$ century. Fourier was concerned that equations should not be affected by the choice of a particular system of units; in order for this to be the case, he said that it is necessary that "every term ... have the same total exponent" with respect to a given quantity [Fourier (1955) art. 161]. Fourier related these "exponents" to a change in the size of the relevant unit, and argued as follows: "suppose ... the unit of length to be changed, and its second value to be equal to the first divided by $m$. Any quantity $x$ which in the equation ... represents a certain line $a b$, and which, consequently denotes a certain number times the unit of length, becomes $m x$, corresponding to the same length $a b \ldots$ thus the dimension of $x$ with respect to the unit of length is 1 " [ibid.].

James Clerk Maxwell developed the concept of dimension considerably in his Treatise on Electricity and Magnetism (first published in 1873). He defined dimension as follows: "When a given unit varies as the $n$th power of one of [the fundamental] units, it is said to be of $n$ dimensions as regards that unit", labelling the fundamental units of mass, length and time as $[M],[L]$ and $[T]$ [Maxwell (1873) art. 2-5]. James Thomson, however, objected to the language used by Maxwell and his contemporary, J.D. Everett, in their treatment of dimensions, arguing that "we have no right to speak of" such concepts as the "square of the unit of time", or hence, generalising, of a "power" of a unit, since a unit is not a number. Instead, Thomson developed Fourier's original definition in terms of a change in size of a fundamental unit, and re-branded $M, L$ and $T$ as "change-ratios" - the ratios by which these units might change. Maxwell's dimensional formulae, such as $M L T^{-2}$ for force, are then understood to represent the corresponding change in a derived quantity such as force [Thomson (1878) p378]. As Daniel Mitchell has pointed out, the emergence of the concept of dimension in the $19^{\text {th }}$ century was closely linked, not only with unit conversion, but also with absolute units and measurement protocols [Mitchell (2017) p3]. 
The weakness of Maxwell's dimensional formulae, in Thomson's interpretation, is the arbitrariness of the factors. What we are really interested in is not $M, L, T$ etc., but the powers to which these quantities are raised. Thus it is tempting to represent dimensions in terms of the powers only, as Fourier did, or as as-an $n$-component vector in an $n$-dimensional space, where $n$ is the number of base quantities [Subramanian et al. (1985); see also Remillard (1983), Mohr (2008), and Moon \& Spencer (1949)]. In this vector format, the dimensions of force in $M L T$ space would be $(1,1,-2)$. The original format is probably better, however, when the base quantities are not the usual ones, as the symbols for the change-ratios remind us of what basis we are working in. We shall use both representations in what follows.

Defining dimension in terms of change-ratio puts dimensional analysis onto a firm footing, because it justifies the algebraic manipulation of dimensions, on the basis that the terms $M, L$, $T$ etc. stand for change-ratios, which are numbers, and hence can be squared, multiplied, divided etc. In the rest of this paper, I will therefore adopt the following definition:

The dimension of a quantity is the factor by which the numerical value of that quantity will increase if the base units are reduced by arbitrary numerical factors, expressed as a product of powers of those factors.

The change-ratio interpretation of dimension is rarely mentioned in the technical literature of the late $20^{\text {th }}$ and early $21^{\text {st }}$ centuries. Instead, we find statements such as that of R.C.

Pankhurst, a metrologist whose principal concern is dimensional analysis and scaling: "the dimensions of an area are described as those of length squared, and those of volume as (length) "3 " [Pankhurst (1964) p13] without explaining how we can square or cube a length, which is not a number but a magnitude. A definition of dimension in terms of change-ratio in a book by G.I. Barenblatt, published in 1996, is one of the very few examples of this in the recent technical literature [see Barenblatt (1996) p3].

As is clear from its definition (see section 2), a base quantity is a member of a "conventionally chosen subset" of quantities; the conventional aspect of this reflects the fact that we may want to choose different sets of base quantities for different applications. To the VIM definition, it is necessary only to add the stipulation that our subset must span the set of quantities under consideration: in other words, it should be possible to express any quantity in terms of the base quantities in a unique way. We shall encounter various sets of base quantities in the remainder of the paper.

Mechanical problems require only three base quantities; others may need more. John Roche, in The Mathematics of Measurement, uses the term "basic reference quantities", listing the six such quantities defined by the Conference Générale des Poids et Mesures (CGPM) in 1954 as "length, mass, time, electric current, thermodynamic temperature and luminous intensity" [Roche p186]. ${ }^{6}$ A seventh - "amount of substance" - was added in 1971.

It is actually the number of base quantities that is important, rather than which ones we choose, subject to some restrictions. For instance, we could decide that density is actually a base quantity (measured using a hydrometer) and define mass as a derived quantity, dependent on prior measurements of volume and density; so our mechanical base quantities are still three in number but are now density, length and time. Base quantities need not be the same as fundamental quantities, but they must be related to them.

${ }^{6}$ The CGPM is, of course, the body charged with the definition and maintenance of the corresponding "concrete base units". 
When transforming from one set of base quantities to another we are said to have performed a change of basis.

\section{Change of Basis}

The process of transforming dimensional formulae under a change of basis can be carried out methodically and elegantly by applying the formalisms of linear algebra. (The terms "basic", "basis" and "base" that one encounters in dimensional analysis and related fields have probably been "borrowed" from that discipline). Various authors have attempted to represent a change of basis using a matrix format, but they have not always distinguished clearly between the transformation of dimensions and that of units or quantities. Of these, only dimensions (conceived as change-ratios) are entities for which multiplication and addition are defined, and hence only they can be represented by vector spaces. [see Subramanian et al. (1985) p238; also Remillard (1983) p137; Mohr (2008) p130; Moon \& Spencer (1949) p172]. No one author has developed a vector-based method of basis transformation that can be used "off the shelf", so we proceed from first principles.

Let us assume that we wish to perform a change of basis from $M L T$ to a basis consisting of momentum, force and velocity, which we could call a $P F V$ space, since the letter $p$ is usually used for momentum.

We know that in the MLT basis, the dimensions of our new base quantities are

$$
\begin{aligned}
& P=M L T^{-1} \\
& F=M L T^{-2} \\
& V=L T^{-1}
\end{aligned}
$$

where we recall that $P$, for example, is the factor by which the numerical value of a momentum will increase if the units of mass, length and time are decreased by factors $\mathrm{M}, \mathrm{L}$, T respectively.

We can write this as

$$
\begin{aligned}
& \log P=\log M+\log L-\log T \\
& \log F=\log M+\log L-2 \log T \\
& \log V=\log L-\log T .
\end{aligned}
$$

or, in matrix format,

$$
\left[\begin{array}{l}
\log P \\
\log F \\
\log V
\end{array}\right]=\left[\begin{array}{lll}
1 & 1 & -1 \\
1 & 1 & -2 \\
0 & 1 & -1
\end{array}\right]\left[\begin{array}{l}
\log M \\
\log L \\
\log T
\end{array}\right]
$$

If the matrix can be inverted, we can write

$$
\left[\begin{array}{l}
\log M \\
\log L \\
\log T
\end{array}\right]=\left[\begin{array}{lll}
1 & 1 & -1 \\
1 & 1 & -2 \\
0 & 1 & -1
\end{array}\right]^{-1}\left[\begin{array}{l}
\log P \\
\log F \\
\log V
\end{array}\right]
$$


and this can be done as long as the determinant

$$
\left|\begin{array}{lll}
1 & 1 & -1 \\
1 & 1 & -2 \\
0 & 1 & -1
\end{array}\right|
$$

is non-zero, and it can be easily established that it is.

Inverting the matrix then gives

$$
\left[\begin{array}{l}
\log M \\
\log L \\
\log T
\end{array}\right]=\left[\begin{array}{ccc}
1 & 0 & -1 \\
1 & -1 & 1 \\
1 & -1 & 0
\end{array}\right]\left[\begin{array}{l}
\log P \\
\log F \\
\log V
\end{array}\right]
$$

whence

$$
\begin{aligned}
& M=P V^{-1} \\
& L=P F^{-1} V \\
& T=P F^{-1}
\end{aligned}
$$

This tells us that, in the new basis, mass, length and time are all derived quantities measured in derived units related to the base units in accordance with the formulae given above; and the dimensions of any other quantity, such as energy, can be found (and hence units chosen) by substituting for $M, L, T$ from the above equation into the usual $M L T$-dimensional formula for that quantity.

Having explained the practice of change of basis, we are now in a position to examine various alternative systems of units that have been postulated.

\section{Natural Units}

The practice of defining units in terms of physical constants belongs to the SI reforms of the late $20^{\text {th }}$ century; however, the idea of doing so is much older. The first attempt was by George Johnstone Stoney, a member of the British Association committee on electrical standards, in 1881. Stoney wanted fundamental units to be independent of any human construction, and instead, based on physical constants. He took the base quantities to be mass, length and time; the problem he faced was that there were no obvious physical constants which could be used as standards of these quantities. However, there were two "mechanical" constants, namely the speed of light $c$ and the gravitational constant $G$. To these he added a third constant, which we know today as the charge on the electron, but in Stoney's day no such particle had been discovered or postulated. Stoney reasoned, from the values of the Faraday constant (the quantity of charge required in electrolysis to produce a mole of a substance) and Avogadro's number (the number of atoms or molecules in a mole) that there was a natural unit of charge $e$, although he did not regard this charge as fundamental because charge was seen as a derived quantity [O'Hara (1975) p269].

To understand how Stoney used this information to define a set of units, we need to transform from an $M L T$ space to a $Q V \Gamma$ space whose base quantities are charge $(Q)$, velocity $(V)$ and 
gravitation $(\Gamma){ }^{7}$ As these quantities are represented in $M L T$ space by the vectors $(1 / 2,3 / 2$, $-1),(0,1,-1)$ and $(-1,3,-2)$ (the first of these corresponding to Maxwell's evaluation of electric charge in the electrostatic system), we can represent the change of basis in the following way:

$$
\left[\begin{array}{l}
\log Q \\
\log V \\
\log \Gamma
\end{array}\right]=\left[\begin{array}{ccc}
1 / 2 & 3 / 2 & -1 \\
0 & 1 & -1 \\
-1 & 3 & -2
\end{array}\right]\left[\begin{array}{l}
\log M \\
\log L \\
\log T
\end{array}\right]
$$

Inverting the matrix, we find that

$$
\left[\begin{array}{c}
\log M \\
\log L \\
\log T
\end{array}\right]=\left[\begin{array}{ccc}
1 & 0 & -1 / 2 \\
1 & -2 & 1 / 2 \\
1 & -3 & 1 / 2
\end{array}\right]\left[\begin{array}{l}
\log Q \\
\log V \\
\log \Gamma
\end{array}\right]
$$

Translating this back into more conventional dimensional format yields

$$
\begin{aligned}
& M=Q \Gamma^{-\frac{1}{2}} \\
& L=Q V^{-2} \Gamma^{\frac{1}{2}} \\
& T=Q V^{-3} \Gamma^{\frac{1}{2}}
\end{aligned}
$$

so that, in the case of length, for example, any quantity which is a charge multiplied by the square root of "gravitation" and divided by the square of a velocity, can be chosen as a unit of length. Clearly the most obvious choice is $e \sqrt{G / c^{2}}$.

Hence we can construct units of mass, length and time from our three physical constants - the Stoney units:

$$
M_{J}=\frac{e}{\sqrt{G}} ; L_{J}=\frac{e \sqrt{G}}{c^{2}} ; T_{J}=\frac{e \sqrt{G}}{c^{3}}
$$

In 1899, Max Planck introduced a similar system of units $M_{P}, L_{P}, T_{P}$, again based on three fundamental constants, but with $e$ replaced by $\hbar$ :

$$
M_{P}=\sqrt{\frac{\hbar c}{G}} ; L_{P}=\sqrt{\frac{\hbar G}{c^{3}}} ; T_{P}=\sqrt{\frac{\hbar G}{c^{5}}}
$$

\section{Duff's Argument}

Michael Duff's paper Comment on Time-Variation of Fundamental Constants, [Duff (2004) p2] was a response to reports of an apparent change in the value of $\alpha$ over cosmological timescales [Webb et al. (1999) p887] and comments by other physicists suggesting that this could be attributed to a variation in one of the constants in terms of which it is defined [see

\footnotetext{
7 "Gravitation" is, however, an odd choice of "quantity" since the gravitational constant -is the only entity with dimensions $(-1,3,-2)$ and hence it is debatable whether "gravitation" is a measurable quantity in the sense of comparing an unknown quantity with a standard of the same kind. On the assumption that a change-ratio for gravitation makes sense, I represent it as $\Gamma$, to distinguish it from the gravitational constant, $G$.
} 
e.g. Davies et al. (2002)]. He believes that for a constant to be truly fundamental it must be dimensionless, and he does not appear to allow for the possibility that a dimensioned quantity can exist independently of any particular system of units. In order to demonstrate what he regards as the absurdity of the suggestions made by Davies et al., he derives three different formulae for the fine structure constant in three different systems of units.

The argument depends on the idea that physical laws and formulae can vary according to which units we are using: they are unit-dependent. The unit systems to which Duff refers each involve choosing three base quantities from electric charge, velocity, action and "gravitation". For each of these base quantities, Duff chooses as a unit a fundamental constant with the appropriate dimensions; the constants are $e, c, \hbar$ and the gravitational constant $G$ respectively. He claims that:

$$
\begin{array}{ll}
\text { "In Planck units }(\hbar=c=G=1) & e^{2}=\alpha \\
\text { In Stoney units }(c=e=G=1) & \hbar=1 / \alpha \\
\text { In Schrodinger units }(\hbar=e=G=1) & c=1 / \alpha "
\end{array}
$$

("Schrodinger units" here being his name for a similar system of his own devising, where the three fundamental constants are clearly $\hbar, e$ and $G$.) $[\text { Duff (2004) }]^{8}$

Hence $\alpha$ can be expressed as $e^{2}, 1 / \hbar$, or $1 / c$ depending on which system of units we choose. This means we must ascribe any observed or postulated change in $\alpha$ to whichever of the other three our units system points us to, since in each case there appears to be only one candidate; and hence, by a sort of reductio ad absurdum, $\alpha$ cannot depend on any of them, and so must be regarded as more fundamental.

He concludes from this that "assigning a change in $\alpha$ to a change in $e$ (Planck) or a change in $\hbar$ (Stoney) or a change in $c$ (Schrodinger) is entirely a matter of units, not physics"- [ibid. p3].

We can see that (9a), (9b) and (9c) appear to follow from substituting the given unit values

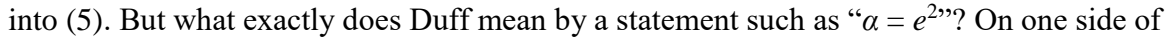
this identity we have a number we know to be dimensionless; on the other, a dimensioned quantity. This is because it is a unit-dependent statement, and so the full wording must be retained, i.e. " $\alpha=e^{2}$ if $e$ is the number of Planck units of electric charge in the charge on the electron", so that $e$ is now a number, not a magnitude. What is the Planck unit of electric charge? In the Planck system, the base quantities are action, velocity and gravitation, and the base units are $\hbar, c$ and $G$. We note that Duff is using the version of Coulomb's Law given in (3), so that the dimensions of charge are $(1 / 2,3 / 2,-1)$ in an $M L T$ quantity space. In an $A V \Gamma$ space, the dimensions of charge can be calculated as follows:

$$
\log Q=\left[\begin{array}{lll}
1 / 2 & 3 / 2 & -1
\end{array}\right]\left[\begin{array}{l}
\log M \\
\log L \\
\log T
\end{array}\right]
$$

Using a similar procedure as for the Stoney units, it can be shown that

\footnotetext{
${ }^{8}$ I have re-formatted Duff's statement slightly to make it clear that my equation numbers refer to the second statement in each line.
}

Commented [ML1]: A small point: "units" isn't part of his name - though it's obvious, a simple change in structure would sort it out.

Commented [WU2]: "Schrodinger uniits" is the name of system of units. It is the name given to that system by Duff - so it is "his name" for that system. Perhaps if this looks unwieldy you is "his name" for that system. Pe
could just delete "his name for"? 


$$
\left[\begin{array}{l}
\log M \\
\log L \\
\log T
\end{array}\right]=\left[\begin{array}{ccc}
1 / 2 & 1 / 2 & -1 / 2 \\
1 / 2 & -3 / 2 & 1 / 2 \\
1 / 2 & -5 / 2 & 1 / 2
\end{array}\right]\left[\begin{array}{l}
\log A \\
\log V \\
\log \Gamma
\end{array}\right]
$$

so that

$$
\log Q=\left[\begin{array}{lll}
1 / 2 & 3 / 2 & -1
\end{array}\right]\left[\begin{array}{ccc}
1 / 2 & 1 / 2 & -1 / 2 \\
1 / 2 & -3 / 2 & 1 / 2 \\
1 / 2 & -5 / 2 & 1 / 2
\end{array}\right]\left[\begin{array}{l}
\log A \\
\log V \\
\log \Gamma
\end{array}\right]=\left[\begin{array}{lll}
1 / 2 & 1 / 2 & 0
\end{array}\right]\left[\begin{array}{l}
\log A \\
\log V \\
\log \Gamma
\end{array}\right]
$$

Hence, in the Planck system, $Q=A^{1 / 2} V^{1 / 2}$ and $e$ is measured in the derived units 9 $\hbar^{1 / 2} c^{1 / 2}$.

Recall that, according to the change-ratio definition of dimension, dimensions $A^{\mathrm{a}}$ indicate that the numerical value of a quantity will increase by a factor $A^{\mathrm{a}}$ if the unit of the quantity decreases by a factor $A$. In the Planck system the unit of action is $\hbar$, so that a decrease in $\hbar$ by a factor $k$ will produce an increase in a quantity of charge by a factor $\sqrt{ } k$, and in the square of a quantity of charge (and hence in $\alpha$ ) by a factor $k$. Exactly the same argument applies to a putative decrease in $c$ by a factor $k$ : this will also produce an increase in a quantity of charge by a factor $\sqrt{ } k$, and in $\alpha$ by a factor $k$. Hence, at least while we are "working in" the Planck units, a change in $\alpha$ can be ascribed, not just to a real change in the electron charge, but equally to a change in Planck's constant or the velocity of light.

The situation is similar if we are "working in" either of the other two systems of units that Duff mentions. In Stoney units, action is a derived quantity with dimensions $Q^{2} V^{-1}$; hence a change in $\alpha$ can be ascribed to a change in either $e$ or $c$ just as easily as to a change in $\hbar$; and the same applies in Schrodinger units. In short, whichever system of units we choose to "work in", we can only conclude that a change in $\alpha$ is due to a change in $e, c$ or $\hbar .{ }^{10}$ There is no contradiction, and no absurdity.

One may object that Duff"s statement that "in Planck units, $e^{2}=\alpha$ " (and other similar statements) is perfectly valid, because it utilises an alternative format for the expression of physical quantities, in which the unit is included in the definition of the quantity, so that we have distinct quantities such as "length-in-feet" and "length-in-metres" whose values are pure numbers. ${ }^{11}$ But in this alternative format, the statement "in Planck units, $\hbar=c=G=1$ " is no more valid than in the more common one; we must say something along the lines of "Planck's constant in Planck units of action = velocity of light in Planck units of velocity = gravitational constant in Planck units of gravitation $=1$ ". This wording would remind the reader to consider possible changes, not just in $e$ but also in the Planck units themselves. The error in Duff's argument comes about as a result of the practice of assigning unity numerical value to a constant and then substituting that value into the equation, so that it

\footnotetext{
${ }^{9}$ Note that here $\hbar$ and $c$ are the names of the Planck units; alternatively, to avoid confusion, we could give them more conventional names, such as "the planck" and "the einstein".

${ }^{10} G$ is not a candidate, because it does not appear in the formula for $\alpha$.

${ }^{11}$ See Ipsen or Kyburg for a discussion of this format [Ipsen (1960) pp25-26; Kyburg (1984) p18].
} 
becomes "invisible", and, more to the point, its units do too. A more careful use of Planck units would include explicit references to the units, e.g. " $c=1$ Planck length per Planck time" rather than just " $c=1$ ".

It should be noted, however, that, as previously indicated, (5) is already in a units-dependent format (it is only true for systems of units in which Maxwell's definition of the unit of charge holds, in other words "absolute" systems of units; it is not true in SI or any other system in which charge or current are regarded as fundamental). So using (5) as our starting point is unsatisfactory; -however, let us, for now, imagine that we are living in a universe in which electrical phenomena are mechanical in origin, and electrical quantities are derived quantities. The argument can easily be extended to the more general case in which the electrical constant has not been suppressed.

We must bear in mind that Nature rejected Duff's paper, and hence I am, in a sense, refuting an argument that has already been refuted. However, looking at the reviewers' responses, as well as the responses of other key players, all of which Duff reproduces in the arXiv version of his paper [Duff (2004) p3], it is clear that there were no criticisms of the particular aspect of his argument that I challenge here. It is therefore entirely appropriate to raise this matter now.

\section{The Origin of Natural Units}

We might reasonably ask to what extent the systems of natural units that Duff refers to are actually used nowadays. As already mentioned, the "Schrodinger units" are an invention of Duff's and hence not often encountered. The Stoney units are little more than a historical curiosity; only the Planck units are still widely known. They are not, of course, used as practical units due to their size; but some theoretical physicists believe that the Planck units define a natural scale that is independent of human dimensions, and at which interesting phenomena (such as the unification of the four types of force) may occur. However there is a supra-set of the Planck units, known simply as "natural units", in which $\hbar=c=1$ (and we do not encounter $G$ ) which are in common use in theoretical physics, particularly in particle physics. Almost all textbooks in quantum field theory (QFT) use this system;- and QFT is the language in which our most fundamental and all-encompassing theory - the Standard Model of particle physics - is written.

The use of this system of units leads authors of QFT texts to claim that all quantities have dimensions which can be expressed as powers of a single quantity, usually energy (measured in giga-electron volts or $\mathrm{GeV}$ ), because quantities having the value 1 are regarded as dimensionless. As with the Stoney and Planck systems, choosing $\hbar$ and $c$ and measuring everything in terms of energy suggests that we have performed a change of basis, in this instance into an $A E V$ space (action, energy and velocity). ${ }^{12}$ Modern particle physicists are generally quite happy to quote a distance or time interval in $\mathrm{GeV}^{-1}$ or "inverse giga-electron volts", although, since in this new basis distance actually has the dimensions $A E^{-l} \mathrm{~V}$ while time has dimensions $A E^{-1}$, other units, representing action and velocity, should of course be present. For example, if our unit of action were, say, the "planck", time intervals should be measured in planck per $\mathrm{GeV}$ and not just $\mathrm{GeV}^{-1}$.

${ }^{12}$ Action, energy and velocity span $M L T$ space; so do action, mass and velocity. 
The use of these natural units can be traced back to Richard Feynman's pioneering work on quantum field theory in the 1940s. [See e.g. Feynman (1948) p1431]. In the literature on quantum electrodynamics and QFT of the 1940s and 1950s we see a gradual increase in the use of natural units, and all modern QFT texbooks use them. They are usually introduced towards the beginning of the book, and sometimes justified on the basis that, since $\hbar$ and $c$ do not appear explicitly in the equations, their absence "simplifies" them. Sometimes this claim is accompanied by an acknowledgement that the use of natural units in QFT is not entirely justifiable; for instance, Halzen and Martin admit that claims about the dimensions of quantities in such a system are made "with a slight but permissible laziness" [Halzen \& Martin (1984)p12]. Sometimes it is claimed that they simplify the calculations - for instance, in their textbook, Mandl \& Shaw state that "no advantage is gained by tediously retaining factors of $\hbar$ and $c$ throughout a calculation" [Mandl \& Shaw (1984) p98]. This was undoubtedly true in the days before computers and electronic calculators, when calculation was not an easy matter and had to be done with slide rules or log tables or (if you were lucky) mechanical calculators. It seems likely that this was the main reason for the adoption of natural units: the simplification they afforded to what could, at that time, be very tedious (to use Mandl \& Shaw's word) number-crunching. This is no longer the case; with calculators and computers which can store the values of fundamental constants, there is really no appreciable advantage to be gained by omitting them from formulae.

Feynman does not appear to justify or reference the use of natural units, but he must have been aware of Arthur Eddington's natural units, since his biographer tells us that Feynman learned general relativity from Eddington's book The Mathematical Theory of Relativity, published in 1923 [Mehra (1994) p88].

In this book, Eddington makes frequent use of another supra-set of the Planck units, in which $G=c=1$. (He does not reference the Planck system, so it is possible that his units are an entirely separate creation, but since he was writing only about 20 years after they appeared, it is tempting to postulate such a link; the practice of writing $c=1$ is, of course, a common, but not universal, feature of textbooks on relativity). For instance, he claims that "the mass of the sun is approximately 1.5 kilometres" [Eddington (1923) p87]. Given his subject, one might imagine that he is expressing some relativistic mixing-up of the concepts of mass and length in the extreme conditions found in stars; but in fact the statement relies on nothing more than a piece of simple Newtonian mechanics: the Earth's orbit around the Sun. What he does is to write down Newton's second law for a body of mass $m$ orbiting another body of mass $M$ at a distance $r$ with velocity $v$ :

$$
\frac{G m M}{r^{2}}=\frac{m v^{2}}{r}
$$

which simplifies to

$$
G M=v^{2} r
$$

If we write $v$ as a dimensionless multiple of $c$, so that $v=\beta c$, we now have an equation containing both $G$ and $c$, so that, on substituting their values, the equation appears to be equivalent to

$$
M=\beta^{2} r
$$

whence, since $\beta$ is dimensionless, $M$ does indeed seem to have the dimension of length; and if you do the maths $\left(\beta \approx 10^{-4}, r \approx 1.5 \times 10^{8} \mathrm{~km}\right)$ it does indeed come out as about $1.5 \mathrm{~km}$. 
Eddington's initial statement that "the mass of the sun is approximately 1.5 kilometres" is not meaningful on its own, however; it requires additional information to qualify it, as does the unit-dependent formula for $\alpha$ quoted earlier. One could say that "the mass of the sun is approximately 1.5 kilometres in a system of units in which $G=c=1$ "; however, this carries an implicit assumption that setting the values of constants to 1 makes them dimensionless an assumption that has been shown to be incorrect in the case of Duff's argument. ${ }^{13} \mathrm{We}$ recall that, having substituted numerical values for quantities in an equation, we must also state the units. Since there are no obvious names for such units, I will invent suitable names purely to illustrate my point. Let us call the unit of velocity, which is the velocity of light in Eddington's system, " 1 einstein", and the unit of gravitation " 1 eddington". Then his statement becomes "the mass of the sun is is 1.5 kilometre einstein ${ }^{2}$ eddington ${ }^{-1}$ ".

Alternatively, we can get rid of the constants by using defining equations. In such equations the quantity being defined takes on units dictated by the definition, and is then regarded as a derived quantity. An example is Newton's Second Law, $-F=m a$. This law, in its original form, said only that force is proportional to the "alteration of motion" (rate of change of momentum, or the product of mass and acceleration). So an algebraic statement of the law would require a constant of proportionality $k$, and would take the form $F=k m a$. The constant $k$ would have dimensions, namely those of force divided by those of mass and acceleration. We would then require four dimensions for mechanics: for instance, mass, length, time and force (or some suitable set of linear combinations of these). Force would have its own units and dimensions, and could not be reduced to those of $M, L$ and $T$. That we do not have such a system today is due to the adoption of the law as a defining equation for force, which reduces the number of mechanical dimensions from 4 to 3 . In general, each defining equation reduces the number of base quantities, and hence of dimensions, by 1 .

Hence we could say, for instance, "the mass of the sun is approximately 1.5 kilometres if we define the time between two events as the distance travelled by light emitted at the first event and detected at the second, and we define the gravitational force between two masses as the product of the masses divided by the square of the distance between them". These two definitions between them would imply that time and mass are both derived quantities with the dimensions of length.

Units are social entities; so are definitions. If we are to make quantitative statements about the world which are meaningful to other people, we must ensure that these statements are either unit-invariant, or refer to a system of units that has been agreed on by the members of the community in which we make our statements, and also that the definitions of the quantities involved have been agreed by all members of that community. The statements made by Duff and Eddington that I have quoted in this paper are not necessarily wrong, as long as the context in which they apply is fully explained.

A slightly different interpretation of Eddington's statement is possible, as suggested by an alternative version of it that he gives elsewhere in the book. Here he describes the -mass of the sun as "in gravitational units 1.47 kilometres" [my emphasis]. This is a different way of representing the mass of the sun; it resembles the alternative method of expressing the value of a quantity referred to in section 7 , where we have a unit as part of the definition of the quantity, which is then given as a pure number. However, in this case, the final word

\footnotetext{
${ }^{13}$ It is not the value 1 that is significant here; if we assigned the value $2, \pi$, or 50,000 to $c$ it would still appear to be a dimensionless number. The error arises from assigning a purely numerical value to an entity which is not a pure number but a magnitude (consisting of a number and a unit)
} 
"kilometres" shouldn't be there; "in gravitational units 1.47 " is sufficient, as long as we know what a gravitational unit is. The answer to that question is not clear; perhaps the unit Eddington had in mind was the "gravitational kilometre", which would be a perfectly respectable unit as long as it were defined somewhere (although it would probably suffer from the same risk of misinterpretation by non-experts as does "light year" as a unit of length). Just as a light year is a unit of length, not time, a gravitational kilometre would be a unit of mass, not length.

\section{Fundamental Constants and Unification}

There is a view, sometimes encountered in the literature, which advocates the abolition of certain fundamental constants, not in order to simplify equations or calculations per se, but because it is felt that science has advanced to a stage at which quantities previously regarded as distinct are really "the same", and hence can now be "unified".

The arguments advanced to support such ideas sometimes involve introducing new defining equations as discussed in section 8 (though rarely is such language explicitly used) whilst sometimes it is just a case of advocating natural units. Often these initiatives appeal to progress made in physics in which entities such as space and time, previously regarded as distinct, are now seen as merely different aspects of "spacetime", so it is argued that the corresponding quantities, length and time, should be regarded as the same; and similarly with mass and energy. These convergences can be achieved, numerically speaking, by setting $c=$ 1. Alternatively, they can also be achieved (in a more permanent sense) by defining distance in terms of time, or mass in terms of energy, or vice versa.

An example of this approach is found in the paper by Duff, Okun and Veneziano, in which the authors debate how many fundamental constants are needed. The assumption made here is that it is intrinsically good to reduce the number of such constants; the authors only differ on how far to go in that process, with Duff advocating "no constants", by which he means no dimensioned constants, since, as already mentioned, he believes that the only true fundamental constants are dimensionless, and he also believes that the act of setting a constant to 1 makes it dimensionless, so that each convergence between previously distinct quantities reduces the number of dimensions by 1 . A similar approach is taken by J.M. LévyLeblond- [see Duff et al. (2002); Lévy-Leblond (1998)]. However, as I have previously argued in the case of Newton's Second Law, a reduction in the number of quantities (and therefore dimensions) really requires new defining equations. This is the line taken by Bernhard Lesche in his paper The $c=\hbar=G=1$ question. [see Lesche (2014) p107].

To go into this topic in more detail would probably take us too far from the theme of this paper, but it is as well to at least mention it. It also throws up an interesting contrast between the views of physicists on this issue and those of philosophers. The physicists (Duff and his co-authors, and Lévy-Leblond and Lesche) all seem very keen on reducing the number of quantities, while the philosophers Henry Kyburg and Brian Ellis are more hesitant.

Kyburg asks, for example, "Why can we not reduce the dimensions of length to those of time by taking the speed of light to be unity and dimensionless?" [Kyburg p176]. Against this view, he regards a defence of length and time as distinct concepts to be "a weak answer", and prefers to argue on the grounds that "our structure of physical magnitudes ... must contain a dimension of speeds" because the speed of light is not the only speed we have to consider [ibid.]. He acknowledges the advantages of simplicity, but goes on to point out that "since it 
is direct comparative discriminations that ultimately affect our lives, and that constitute the predictive observational content of our bodies of knowledge, we want to retain distinct dimensions for quantities that admit of distinct comparative judgments" [Kyburg (1984) p175].

Brian Ellis argues along similar lines: "we can only get out of our dimensional formulae what we put into them. And, if we choose to express our laws with respect to particular scales, we shall inevitably impoverish our dimensional formulae" [Ellis (1966) pp147-148]. Ellis has previously identified the concept of "dimension" with "the names of particular classes of similar scales for the measurement of quantities" [ibid. p139], and a scale is a set of multiples of a unit; so what he is saying here is that to tailor our formulae to particular units constitutes impoverishment.

\section{SI: A step towards unit-invariance?}

The SI system incorporates a definition of the ampère which requires a constant to be inserted into Coulomb's Law. This was the same constant that had been proposed by Rücker in 1889 and recommended in 1932 by a Commission of the International Union of Pure and Applied Physics. When the law, and expressions derived from it, are written algebraically, they are, in effect, unit-invariant. The definitions of the electrical vectors, and consequently the format of electrical equations, including the relevant constants, that arose out of that meeting appear not to have made it into the textbooks until the introduction of- the SI system 30 years later. For that reason, the two appear to have been inextricably linked in the minds of many physicists. The unit-invariant equations are seen as simply "SI equations".

Here is a typical example from a modern electromagnetism textbook: after stating Coulomb's Law for the force $F$ - between charges $q_{1}$ and $q_{2}$ a distance $r$ apart as

$$
F=\frac{q_{1} q_{2}}{4 \pi \varepsilon_{0} r^{2}}
$$

the authors describe the equation as being "in SI units" [Grant \& Phillips (1999)p3]. But there is nothing about this equation that limits it to SI, since $\varepsilon_{0}$ can take any value, including $8.85 \times 10^{-12}$ farad metre ${ }^{-1}-(\mathrm{SI})$, or $1 / 4 \pi$ statcoulomb $^{2} \mathrm{dyne}^{-1} \mathrm{~cm}^{-2}$ (cgs). Unfortunately, the subtleties implied by this have not been taken up by the physics community; rather than concluding that SI is just one of a number of unit systems that require $\varepsilon_{0}$ to be explicitly included in the law, it is instead generally assumed that the presence of this symbol means that the formula only works in SI, and hence it is taken as synonymous with the numerical value given above, rather than simply a constant to which units have not yet been assigned, as is the case with the acceleration due to gravity, $g .{ }^{14}$

When one looks beyond degree-level textbooks into more specialised sources and research papers, the incidence of formulae that are effectively, if not nominally, unit-invariant diminishes. Hence it is no surprise that very few authors, even nowadays, define $\alpha$ in the format given in (6). In one of the few papers where this definition can be found, the authors state that

${ }^{14}$ For this reason it might be better to revert, in the case of electrostatic formulae, to the notation used by Rücker and Williams, namely $1 / k$ instead of $1 / 4 \pi \varepsilon_{0}$. 


$$
\alpha=\left(\frac{\mu_{0} c^{2}}{4 \pi}\right)\left(\frac{e^{2}}{\hbar c}\right)
$$

(this is equivalent to the definition given in (6), since $c^{2}=1 / \mu_{0} \varepsilon_{0}$ ) but clearly feel the need to justify this definition: "We use SI units throughout because the as-maintained electrical units are expressed in that system" [Taylor et al.(1969) p381]. Note that, once again, the authors have identified the symbol $\mu_{0}$ (and implicitly $\varepsilon_{0}$ ) with the SI system rather than regarding it as a constant whose numerical value varies with choice of units. The idea that electrical laws are inherently unit-specific is clearly alive and well: an earlier version of this paper was rejected on the advice of a reviewer who cited "the familiar fact that, when electromagnetic quantities are involved, the form in which equations are written depends on choice of units."

Even in textbooks, the new formats of equations took their time to become established. In a book published in 1964 (shortly after the inception of SI) Pankhurst included a fourth dimensional constant (which he wrote as $1 / \varepsilon$ ) -in electrical equations; yet this idea was slow to catch on. Thirty years later, a general physics textbook was published which included the statement that "All measurements can be reduced ultimately to the measurement of length, time and mass. Any physical quantity, no matter how complex, can be expressed as an algebraic combination of these three basic quantities" [Fishbane, Gasiorowicz \& Thornton (1996), p. 9].

Why have physicists been so slow to adopt the new formats? Perhaps because they have been linked so intimately with the SI system, and that system, in turn, was introduced in a very slow and permissive way which allowed those who had "grown up" with the cgs system to simply carry on using it. Hence the old picture of electricity as having mechanical dimensions persisted. SI was seen by many as simply another system of units, the use of which was merely optional.

Thomas Kuhn said in The Structure of Scientific Revolutions that, after a scientific revolution, superseded theories and ideas will wither away, as any remaining supporters die off and textbooks get rewritten [Kuhn (1996) p137]. In saying this, however, he omitted one very important factor - the autonomy of research groups and the pressure on junior members of such groups to conform to the conventions of their superiors. What that means in the case of SI - which might not quite qualify as a "scientific revolution" as such but certainly incorporated some major changes - is that students may well get to degree level having been thoroughly indoctrinated in unit-invariant formats, thanks to those rewritten textbooks, but then find that at postgraduate level there is a lot of pressure to revert to the methods and standards of a previous generation. Thus not only were many of today's most senior physicists educated under a system in which unit-invariance was not seen as an option; they have passed on this view to the next generation, who in turn will pass it on to the next. Since the form of equations constitutes the language of physics, it is understandable that many physicists have preferred to perpetuate the language in which they were taught rather than to embrace the challenge of translation. Hence the idea that the laws of physics must be written in a particular system of units persists. ${ }^{15}$

\footnotetext{
${ }^{15}$ When studying for my PhD in 2003 - many years after the birth of SI - I was put under considerable pressure to quote distances in centimetres and magnetic fields in gauss, despite the measurements we made being many orders of magnitude away from their respective units. I resisted, but with some discomfort.
} 
It is not unusual, of course, for a particular research community to have its own mathematical "language" in which its members communicate. The difficulty arises when a theory has to be explained to someone outside that community. In unit-invariant notation the constants $h$ and $c$ help to identify the dimensionality, and therefore the physical nature, of a particular term; in natural units, this is not possible, because the familiar "mileposts" have disappeared.

In 1999, the editor of the American Journal of Physics, Robert Romer, wrote a provocative editorial entitled "Units - SI Only, or Multicultural Diversity?" [Romer (1999) p13-14]. The editorial begins by quoting the journal's policy that "authors are encouraged to use SI units. Use of SI units is not mandatory, however, if other units are more appropriate."- The editorial provoked a huge postbag of responses which were printed in subsequent editions of the journal, expressing a diversity of opinions on whether or not physicists, and particularly physics teachers, should use SI units or a variety of units. What is particularly interesting about this exchange is that it was not limited to a discussion of units, but also covered the forms of certain equations and the definitions of certain quantities, wandering seamlessly from the one topic to the other - evidence that the currently accepted formats for physical laws are regarded as somehow being part of the SI system. Included in the editorial, for instance, was the statement that "I like to see the velocity of light in Maxwell's equations" and one of the respondents argued that the vectors $\mathbf{B}$ and $\mathbf{H}$ ought to be measured in the same units, as should $\mathbf{D}$ and $\mathbf{E}$. Another, the eminent particle physicist Val Fitch, expressed the view that "any system that gives $E$ and $B$ different units, when they are related through a relativistic transformation, is on the far side of sanity...- ${ }^{16}$

None of those who participated in this discussion deviated from the view that the format of a physical equation is dictated by one's choice of units; none made any reference to the unitinvariant option, by that or any other name. But the main factor determining the format of an equation is the definitions of the quantities involved, which in the case of electromagnetism, were determined, not by the SI system - which was introduced in 1960 - but, as one correspondent, R.G._Chambers, pointed out, -by the 1932 IUPAP Commission referred to previously.

At this meeting, according to E. Griffiths (who was its secretary), the relationship between the electromagnetic vectors was discussed at length, and the Chairman, Sir Richard Glazebrook, is reported as having voiced his recollection of Maxwell's view on this from when he (Glazebrook) was Maxwell's student; so the discussion could be said to have been an extension of that going on in Maxwell's time: it was thus a debate that took place long before the introduction of the SI system. However, even in the Report of this meeting, no clear distinction was made between the questions of how we define physical quantities, and of what units we measure them in.

The concept of "multicultural diversity", as applied to unit choice, fits very well with the idea of unit-invariance. If we have equations which are valid in all systems of units, those who prefer a given system of units can easily adapt them to their own preferences by substituting the appropriate numerical values (including, in some cases, numerical values that are equal to

${ }^{16}$ This was no doubt supposed to appeal to the many readers who, like Fitch, were familiar with relativistic transformations that link pairs of quantities such as distance and time. But his argument only makes sense if one is using natural units, where no clear distinction can be made between distance and time if $c=1$. Outside this domain, there can be few physicists who would suggest that we measure different quantities in the same units. 
unity). Yet not only did none of the participants in the discussions - in Paris or in the pages of the $A J P$ - appear to acknowledge this option, but I am not aware of it having been suggested anywhere or anywhen else, aside from the handful of quotations by Fourier, Maxwell, Rayleigh etc already cited. Even in the report of the Coulomb's Law Committee of the American Association of Physics Teachers in 1950 - described by Chambers as "a model of clear thinking" - many of the key equations appear in pairs, and a footnote proclaims that these equations are "written in both Gaussian and mksc units" [Chambers (1999) p469; Coulomb's Law Committee (1950) p6]. The only difference is that one of each pair contains the constant $1 / 4 \pi \varepsilon_{0}$ while the other does not; quite why the former could not do duty for both systems of units, with $\varepsilon_{0}$ taking different numerical values, is not clear, especially when other constants such as $g, G, h$, etc are commonly regarded as having different numerical values in different systems of units.

\section{Conclusion}

In this paper, we have found that, although prominent individuals and bodies have espoused unit-invariance from the 1820 s up to at least the 1960 s, unit-dependent representations are still quite common. We also discovered that arguments based on unit-dependent formulae may lead to incorrect conclusions; that unit-dependent formulae have been promoted in the interests of simplicity, particularly in the pre-computer age, to simplify calculations; and that this has been at the expense of generality. We have noted that the SI system provides a framework that is conducive to unit-invariance, but that this aspect of the system has not been taken up, partly due to a misunderstanding of the nature of modern electromagnetic formulae, and partly because of the nature of postgraduate training.

So - should physical laws be unit-invariant? Well, it is not the rôle of the philosopher to tell scientists what they should do; formats and conventions can only be agreed by consensus in the scientific community itself, through bodies such as the IUPAP committee referred to in this paper to determine the formats of electromagnetic equations. On the other hand, philosophy of science provides the critical dimension that has almost disappeared from "hard" science since the days of "natural philosophy", and it is surely right to hold scientific practices up to scrutiny.

I am not suggesting that quantum field theory should be re-written in a unit-invariant format. In any case, physicists who use the theory are generally aware that the formulae are only valid in a special system of units, and that, if they need to calculate some quantity in more conventional units, they will have to "translate" them back into a unit-independent format by inserting the constants $c$ and $\hbar$ by inspection, to restore the correct dimensionality. It is at the learning stage that natural units present a particular problem; in my ewn-personal experience, since the constants carry information about the dimensions of the various terms, they help the reader to understand the physical interpretations of formulae which would otherwise be purely mathematical expressions. Hence their absence makes learning a very difficult theory even harder.

Nevertheless, the assertion, often encountered in QFT texts, that a magnitude may simply be replaced by a dimensionless number, is clearly false, and should be challenged; and if formulae such as the "cgs" version of the definition of the fine structure constant are used in isolation, it should surely be stated clearly that they are only valid in a specific system of units. It would also help if authors acknowledged that many formulae in common use in physics, although currently described as being "in SI units", are in fact unit-invariant. 
I would like to thank the members of the Philosophy and History of Physics Reading Group in the Department of History and Philosophy of Science, Cambridge University, and Professor Hugh Mellor, for their helpful comments; also to Dr Michael Jewess for the Royal Society reference.

\section{Funding}

This research did not receive any specific grant from funding agencies in the public, commercial, or not-for-profit sectors.

\section{References}

Barenblatt, G.I. (1996). Scaling, self-similarity, and intermediate asymptotics. Cambridge University Press.

Bridgman, P. (1922). Dimensional Analysis. Yale University Press.

Campbell, N. (1928). An Account of the Principles of Measurement and Calculation. Longman.

Chambers, R.G. (1999) Units - B,H,D and E. Am.J. Phys. 67(6) 469.

Coulomb's Law Committee (1950). Teaching of Electricity and Magnetism at the College Level. Am. J. Phys. 18(1) 1-25.

Davies, P.C.W., Davis, T.M., and Lineweaver, C.H. (2002). Black holes constrain varying constants. Nature 418 pp 602-3.

Dicke, R.H. (1962). Mach's Principle and Invariance under Transformation of Units. Phys. Rev. 125 (6) 2163-7.

Duff, M.J. (2004). Comment on Time-Variation of Fundamental Constants, arXiv:hep-th/0208093v3.

Duff, M.J., Okun, L.B., and Veneziano, G. (2002). Trialogue on the Number of Fundamental Constants, arXiv:physics/0110060v3.

Eddington, Sir A. (1923). The Mathematical Theory of Relativity. Cambridge University Press

Ellis, B. (1966). Basic Concepts of Measurement. Cambridge University Press

Feynman, R. (1948). Relativistic Cut-Off for Quantum Electrodynamics, PhysRev 74 (10)

Fishbane, Gasiorowicz \& Thornton (1996). Physics for Scientists \& Engineers. Prentice Hall.

Fourier, J. (1955). Analytical Theory of Heat. Dover.

Grant, I. \& Phillips, W. (1999). Electromagnetism (2 ${ }^{\text {nd }}$ ed.). Wiley.

Griffiths, E. (1932). Electrical and Magnetic Units. Nature 130, pp 987-989.

Halzen, F. \& Martin, A. (1984). Quarks and Leptons. Wiley.

Ipsen, D.C. (1960). Units, Dimensions \& Dimensionless Numbers. McGraw-Hill.

Kuhn, T. (1996). The Structure of Scientific Revolutions ( $3^{\text {rd }}$ ed.) University of Chicago Press.

Kyburg, H. (1984). Theory and Measurement. Cambridge University Press.

Lesche, B. (2014). The $c=\hbar=G=1$ question. Studies in the History \& Philosophy of Modern Physics. 47, 107-116.

Lévy-Leblond, J-M (1998). Dimensional Angles and Universal Constants. Am.J.Phys. 66 (9) pp814-815.

Macagno, E. (1971). Historico-Critical Review of Dimensional Analysis. Journal of the Franklin Institute 292 (6), 391-402.

Mandl, F., \& Shaw, G. (1984). Quantum Field Theory. Wiley.

Maxwell, J.C. (1873) A Treatise on Electricity and Magnetism. Oxford.

Mehra, J. (1994) The Beat of a Different Drum: The Life and Science of Richard Feynman. Oxford University Press. 
Mitchell, D.J. (2017), Making Sense of Absolute Measurement. Studies in History and Philosophy of Modern Physics. 58, 63-79.

Mohr, P. (2008). Defining Units in the Quantum Based SI. Metrologia 45, 129-133.

Moon, P., and Spencer, D.E. (1949).The Dimensions of Physical Concepts. Am. J. Phys. 17, 171

O'Hara, J. (1975) George Johnstone Stoney FRS and the Concept of the Electron. Notes and Records of the Royal Society of London, 29, No. 2, pp. 265-276.

Pankhurst, R.C. (1964). Dimensional Analysis and Scale Factors. Chapman \& Hall. Riordan, S. (2015). The Objectivity of Scientific Measures. Studies in the History and Philosophy of Science, 50, 38-47.

Roche, J. (1998). The Mathematics of Measurement. Athlone.

Romer, R. (1999). Units - SI-only, or Multicultural Diversity? Am. J. Phys. 67(1) 13-16.

Royal Society (1969). Symbols, signs, and abbreviations recommended for use in British scientific publications.

Rücker, A. (1889). On the Suppressed Dimensions of Physical Quantities. Phil. Mag. series 5, 27, 104-114.

Remillard, W. (1983). Applying Dimensional Analysis. American Journal of Physics 51, 137-140.

Sommerfeld, A. (1922). Atomic Structure and Spectral Lines. Translated by H.L.Brose 1923. Methuen, London.

Subramanian, P., Gnanapragasam, B., and Janhavi, G. (1985), Vector Methods in Dimensional Analysis. Eur. J. Phys. 6, 238-241.

Taylor, B., Parker W., \& Langenberg, D. (1969) Determination of $e / h$. Rev. Mod. Phys. 41 (3) 375-496.

Thomson, J (1878). On Dimensional Equations, \& on some Verbal Expressions in Numerical Science. In Thomson, (1912), Collected Papers in Physics and Engineering. Cambridge University Press.

VIM (2008). Vocabulaire international de métrologie. BIPM.

Webb, J.K., et al. (1999). Search for Time Variation of the Fine Structure Constant. Phys. Rev. Lett. 82 (5), pp 884-887. 